\title{
TIEMPO DE COMPROMISO MOTOR EN EDUCACIÓN FÍSICA PARA ENSEÑANZA PRIMARIA. UNA REVISIÓN SISTEMÁTICA
}

\author{
Motor Commitment Time in Physical Education for Elementary School.
}

\author{
A Systematic Review
}

\author{
Alixon Reyes Rodríguez ${ }^{1}$ \\ ORCID: 0000-0001-9857-0930 \\ Mario Ibañez Alarcón ${ }^{2}$ \\ ORCID: 0000-0002-9175-8145 \\ Nathan Villagra ${ }^{3}$ \\ ORCID: 0000-0001-5280-5802 \\ Pablo Maureira ${ }^{4}$ \\ ORCID: 0000-0002-6012-1205 \\ Gustavo Pávez-Adasme \\ ORCID: 0000-0003-4377-384X \\ 12345 Universidad Adventista de Chile \\ Correspondencia: alixdavid79@gmail.com \\ Recibido: 30/05/2021
}

Aceptado: 09/09/2021

Resumen: El tiempo de compromiso motor (TCM) tiene implicaciones relevantes en la clase de Educación Física, dado que está relacionado con la actividad física regular que debe realizar un niño o adolescente y las sugerencias de organizaciones internacionales en relación con la mínima cantidad requerida para el mantenimiento de la salud. Este trabajo tiene como objetivo analizar el tiempo de compromiso motor en clases de Educación Física en enseñanza primaria a partir de una revisión sistemática. La búsqueda se hizo en Web of Science, Scopus, ERIC y Dialnet, seleccionando artículos publicados entre 2009 y el primer semestre de 2021. Aplicando criterios de inclusión y exclusión, fueron seleccionados ocho artículos que comprenden una población de estudio de 1262 alumnos. El TCM es bajo en las clases observadas, y la actividad física de moderada a vigorosa presenta datos a la baja. En el estudio de mejor resultado, el TCM alcanza el 46,67 \% y, en los restantes, 43,44\%, 42,8 \%, 41,45\%, $32 \%, 31,5 \%, 28,53 \%$ y 18,10\%. Los niños logran mayor TCM que las niñas; niños y niñas en las primeras edades logran mayor participación y tienen más actividad física; el horario matutino y algunas unidades didácticas favorecen un mayor TCM. Se constata que el TCM es bajo en las clases de Educación Física, y que la gestión del tiempo en la clase condiciona la magnitud de la actividad física que realizan niños y niñas. El tiempo de compromiso motor alcanzado en la clase es insuficiente para cubrir las sugerencias mínimas de actividad física en el período escolar.

Palabras clave: compromiso motor; tiempo activo; tiempo efectivo; educación física; enseñanza primaria; actividad física; sedentarismo. 
Abstract: Motor commitment time (MCT) has relevant implications in the Physical Education class, since it is related to the regular physical activity that a child or adolescent should perform and the suggestions of international organizations in relation to the minimum amount required for the maintenance of health. The aim of this work is to analyze the engagement motor time in physical education classes in primary education through a systematic review. The search was made in Web of Science, Scopus, ERIC and Dialnet, selecting articles published between 2009 and the first semester of 2021. Applying inclusion and exclusion criteria, eight articles were selected, comprising a study population of 1262 students. The MCT is low in the classes observed, and moderate to vigorous physical activity presents declining data. In the study with the best result, the MCT reaches $46.67 \%$ and, in the remaining studies, $43.44 \%, 42.8 \%, 41.45 \%, 32 \%, 31.5 \%, 28.53 \%$ and $18.10 \%$. Boys achieve higher MCT than girls; boys and girls in the early ages achieve higher participation and have more physical activity; the morning schedule and some didactic units favor a higher MCT. It was found that the MCT is low in Physical Education classes, and that time management in the class conditions the magnitude of the physical activity carried out by boys and girls. The motor engagement time achieved in the class is insufficient to cover the minimum suggestions for physical activity in the school period.

Keywords: motor commitment; physical education; elementary school; physical activity; sedentary lifestyle.

\section{Introducción}

El tiempo de compromiso motor (TCM) es un indicador que viene siendo desarrollado originalmente en los estudios de Carroll \& Spearrit (1967), Pieron (1988) y Siedentop (1998), y contempla la cantidad de tiempo en que los estudiantes realizan actividad física (AF) intencionada en la clase de Educación Física (EF; Reyes et al., 2020). Para ello no cuenta el tiempo de gestión para organización de la clase, tampoco el tiempo de espera de indicaciones del profesor para el desarrollo de actividades específicas ni el tiempo en el que esperan su turno para ejecutar la acción motriz requerida. Así, el TCM implica la magnitud de la dimensión temporal en la que los estudiantes ejecutan de manera concreta una acción motriz requerida, sea correr, ejecutar pasos en una acción dancística, adopción de posiciones (de pie, sentado, de cuclillas, apoyo facial, apoyo dorsal, apoyo invertido, decúbito abdominal, decúbito dorsal, etc.), saltos, caminata, gateos, reptación, trepas, entre otros patrones de movimiento.

Hablar del TCM implica además considerar otros rangos de tiempo que pueden señalarse en la clase de EF, entre ellos, tiempo de clase, tiempo real de la clase, tiempo empleado en la tarea (Olmedo, 2000). La señalación de estos tiempos es de relevancia, dado que se trata precisamente de la organización docente, de la gestión del tiempo en la clase para el logro adecuado de los objetivos correspondientes, de la administración didáctica en la sesión. De acuerdo con Gracia y Ruiz (2017) y Olmedo (2000), el tiempo de clase señala el destinado por el programa propio de la arquitectura curricular; el tiempo real de clase implica aquel que finalmente emplea el docente en clase de forma concreta, más allá de las disposiciones 
curriculares, y el tiempo empleado en la tarea es aquel que emplea el estudiante en la resolución de una tarea en particular. Pero también sucede que en la clase se emplea tiempo para algunas otras disposiciones didácticas como la asignación de tareas, la explicación, la demostración, la organización de grupos, entre otras.

Considerar el TCM en la clase de EF implica atender un tema relevante como la gestión del tiempo en la clase, y eso tiene que ver necesariamente con el tiempo que la escuela destina para que los estudiantes realicen AF en un tiempo organizado e intencionado en la arquitectura curricular (Fernández, 2019; Reyes et al., 2020). Este tiempo, además, se ve reflejado en los programas de la asignatura según niveles educacionales. Estos temas guardan vinculación debido a que la inactividad física se ha mantenido como la cuarta causa de muerte en el mundo (Celis-Morales et al., 2020; Márquez, 2020) y uno de los factores de riesgo más relevantes en la proliferación de enfermedades crónicas no transmisibles (ECNT), tanto en niños como en adolescentes y adultos (Carrillo, 2020; Chávez et al., 2018; Salas et al., 2018a).

Esto preocupa mucho más al hablar de niños y adolescentes, por encontrarse estos en una etapa clave para formar hábitos de vida activa y saludable (Cavill et al., 2016). En este contexto, Chile se encuentra en el penúltimo lugar entre 49 países estudiados en lo que se refiere a la AF en niños y jóvenes (Aubert et al., 2018). En Chile, solo tres de cada diez niños menores de cinco años realizan por lo menos tres horas de AF al día, mientras que solo dos de cada diez adolescentes registran una hora al día (Aguilar-Farías et al., 2017). En cuanto a los niveles de AF en el contexto escolar, solamente entre el 12,8\% y $33 \%$ de los estudiantes chilenos participan tres o más veces por semana en la clase de EF y Salud, mientras que solo uno de cada cuatro estudiantes presenta refuerzo positivo, repitiendo el comportamiento a modo de ser físicamente activo (Universidad de la Frontera \& Active Healthy Kids Global Alliance, 2018). Este mismo estudio revela que el $80 \%$ de los niños y niñas en edad escolar son físicamente inactivos. A medida que los niños crecen, realizan menos AF (Guthold et al., 2019), y vale destacar que, en 2016, la prevalencia de inactividad física en niños entre 11 y 17 años estaba en el orden de $84,2 \%$ en Chile, y en niñas, en un 91,2 \%. El tema se hace más preocupante dadas algunas medidas tomadas por el Ministerio de Educación (MINEDUC) y el Consejo Nacional de Educación (CNED) que apuntan a reducir la actividad física en estas poblaciones. Nos referimos en particular a la medida de suprimir la obligatoriedad de la Educación Física en 3. y 4. ${ }^{\circ}$ medio (Consejo Nacional de Educación de Chile, 2019; Ministerio de Educación, 2019). La $\mathrm{EF}$ se ha vuelto una asignatura prescindible en muchos lugares, y en Chile el tiempo 
destinado a esta asignatura es cooptado por cualquier otra actividad que se necesite realizar en la institución (Vio del Río, 2019).

Sumado a lo anterior, cabe señalar el estado nutricional de los preescolares y escolares de establecimientos municipalizados y particulares subvencionados de Chile. Datos suministrados por la Junta Nacional de Auxilio Escolar y Becas (JUNAEB; 2018) evidencian que, en los niveles de prekínder, kínder y primero básico, el sobrepeso y la obesidad superan el estado nutricional normal, mientras que en $1 .^{\circ}$ medio el sobrepeso y la obesidad alcanzan un 46,2 \%. El estudio Radiografía de la Obesidad Infantil de 2020 destaca que la obesidad creció en Chile en un 66,3\% entre 2005 y 2018 en niños menores de seis años; que entre 1997 y 2018 el sobrepeso y la obesidad en niños que cursan $1 .^{\circ}$ básico se incrementó en un 50,9 \% y que en niños y niñas de $1 .^{\circ}$ medio se incrementó en un 46,4 \% entre 2009 y 2018 (Ministerio de Desarrollo Social y Familia, 2020). En función de estos datos, puede concluirse que en edades tempranas se es más vulnerable a padecer sobrepeso u obesidad, y esto se encuentra relacionado con la inactividad física (Trejo et al., 2012).

La práctica de AF diaria recomendada por la Organización Mundial de la Salud (2019; Bull et al., 2020; Fondo de las Naciones Unidas para la Infancia, 2019; Organización Panamericana de la Salud, 2019), que corresponde a 60 minutos de actividad física moderada a vigorosa (AFMV), tiene efectos beneficiosos para la salud y contribuye en la prevención de ECNT, como infartos de miocardio, accidentes cerebrovasculares, diabetes, que están relacionadas con el sobrepeso y la obesidad (Leiva et al., 2018; Rodríguez et al., 2020). Además, la AF interviene en la salud mental, mejora significativa de síntomas de depresión, reducción de la ansiedad, aumento de la autoestima, funcionamiento cognitivo, rendimiento académico, entre otros (González y Portolés, 2014; Marques et al., 2017). Pero, al mismo tiempo, "no cumplir con las recomendaciones de actividad física se asocia a mayores niveles de obesidad, diabetes, hipertensión y síndrome metabólico en población chilena" (Díaz-Martínez et al., 2018, p. 585).

En Chile, el Ministerio de Educación (2020) ha generado las Orientaciones para la actividad física escolar, en las que se adhiere a las declaraciones de la OMS (Bull et al., 2020) y reconoce que, en la actualidad, niños y niñas entre $1 .^{\circ}$ y $4 .^{\circ}$ básico, ocupan apenas un 8,5\% del tiempo que están en los establecimientos educacionales en hacer AF, magnitud temporal que es insuficiente y no alcanza para cubrir las demandas mínimas de actividad al día. Entre $5 .^{\circ}$ y $6 .^{\circ}$ básico es menor el tiempo, apenas un 4,25\%. Varios autores señalan que la 
responsabilidad no puede ser adjudicada a la escuela de forma exclusiva, sino que se trata de una labor colectiva en la que, además, debe estar involucrado el entorno familiar (Blanco et al., 2020; Rodríguez et al., 2020; Salas et al., 2018b).

De acuerdo con información suministrada por La Tercera recogida por Chile Vive Sano (“Preocupante realidad”, 2014), un estudio realizado por el Instituto de Nutrición y Tecnología de los Alimentos (INTA) de la Universidad de Chile en clases de EF en tres regiones del país reveló que "de una clase de una hora y media, un niño se mueve constantemente solo 14 minutos en promedio". Así, se ha visto que los estudiantes realizan bajos niveles de AF moderada o vigorosa en la clase de EF (Bevans et al., 2010; Coe et al., 2006; Scruggs et al., 2003), debido a diferentes factores como el contexto del colegio, las características técnicas del profesor, el tamaño del grupo curso, los materiales, las instalaciones deportivas, la duración de la clase y el número de clases por semana (Martins et al., 2016; Smith et al., 2014; Sutherland et al., 2016), entre otros.

Según datos de investigación existe un limitado TCM, es decir, el tiempo que el alumno dedica a la práctica de AF efectiva a determinadas intensidades, en función del objetivo de la clase de EF, es reducido. Esto sucede porque el tiempo de la mayoría de las clases es destinado a las tareas de gestión, tales como pase de asistencia, organización de materiales y el orden dentro de la clase (Bevans et al., 2010), sin una organización y administración adecuadas del tiempo. Esto es un problema, debido a que muchos niños, generalmente provenientes de entornos menos favorecidos, solo cuentan con la clase de EF como instancia para realizar AF (Organización de las Naciones Unidas para la Educación, la Ciencia y la Cultura, 2015) e “incluso, algunos nunca han recibido una clase de Educación Física” (López de D’Amico, 2019, p. 34). Una proporción importante de niños — aún indefinida — adopta un estilo de vida sedentario que se inicia en la infancia, se desarrolla en la adolescencia y alcanza un nivel más crítico en la adultez, en detrimento progresivo de la salud (García et al., 2012; Márquez et al., 2006).

Dados estos antecedentes, surgen preguntas de investigación en relación con las evidencias científicas, que permiten analizar el problema con mayor profundidad. Las preguntas que orientan este trabajo son: ¿cuánto es el tiempo de compromiso motor en la clase de EF en enseñanza primaria?, ¿el TCM en la clase de EF en enseñanza primaria tributa de manera positiva para el cumplimiento de las recomendaciones mínimas de AF para niños y niñas en la semana? 
Este trabajo comparte lo que señala Torres (2011): "La revisión bibliográfica realizada sobre los resultados de investigaciones relacionadas con el tiempo en la EF posibilita establecer los fundamentos teórico-metodológicos para una acertada utilización del tiempo en las clases”, y más cuando en Chile se ha encargado a la EF la enseñanza y práctica de la AF desde el punto de vista epidemiológico (Rodríguez-Rodríguez et al., 2016). Así, el objetivo de este trabajo es analizar el TCM que alcanzan los estudiantes en las clases de EF en enseñanza primaria, a partir de la evidencia científica rastreable.

\section{Materiales y métodos}

Una revisión sistemática es una síntesis de la evidencia científica existente referente a un tema o interrogante específica (Sánchez-Meca y Botella, 2010). Considera el acopio de investigaciones originales y estudios primarios, sistematizando información, filtrando y organizando para sintetizar, evaluar e interpretar la información realmente relevante (Ferreira et al., 2011).

Para efectos de esta investigación, se realiza una revisión sistemática de artículos originales sobre el TCM en las clases de EF que van desde el primer año hasta el sexto año de educación primaria (grupo etario entre 6 y 11 años de edad). Para ello se han cotejado criterios aplicables del procedimiento PRISMA (Urrútia y Bonfill, 2010). La búsqueda se hizo en la base de datos ERIC, el repositorio Dialnet, los índices de Scopus y Web of Science. Las palabras clave fueron: 'tiempo efectivo', 'compromiso motor', 'tiempo activo', con su correlato en inglés, a saber: effective time, motor commitment, active time. Se trabajó con filtros comunes en los índices, el repositorio y la base de datos (comillas, rango temporal, tipo de documento, idiomas). En el caso de las palabras clave 'effective time' y 'active time', estas fueron entrecomilladas y asociadas a la palabra 'physical education' a partir del operador booleano 'and'. Esto, por cuanto se trata de términos con una polisemia y amplitud semántica considerable. Los criterios de inclusión fueron: artículos de revistas en idioma inglés o español, publicados entre 2009 y junio 2021, investigaciones con población de estudio en el contexto educacional, de tipo experimental realizadas por docentes de EF, con clases que también debían ser realizadas por docentes de EF. Los criterios de exclusión fueron: artículos duplicados, trabajos en los que la población perteneciera a los niveles de educación parvularia y/o enseñanza media, artículos en los que no se describe la metodología en el resumen. Los datos considerados 
para el análisis de los artículos fueron: título, autores, año de publicación, país del estudio, objetivo de investigación, población de estudio, TCM constatado en la clase de EF, instrumentos empleados para la medición, resultados y conclusiones.

\section{Resultados}

Ya determinado el volumen de literatura, se hallaron 974 artículos: 76 en ERIC, 370 en Dialnet, 298 en Scopus y 230 en Web of Science. Del total inicial se excluyeron 860 artículos por títulos y resumen, 95 por duplicaciones y acceso restringido. Luego se excluyeron 11 trabajos por metodología y población, quedando un total de ocho documentos (4 en Dialnet, 1 en Scopus y 3 en Web of Science). En primera instancia se observa que la población objeto de estudio en la suma de los artículos seleccionados fue de 1262 estudiantes de enseñanza primaria. Además, estos ocho trabajos seleccionados tienen participación de 29 autores, con un promedio que llega ligeramente a los cuatro autores por trabajo.

Los trabajos fueron publicados en cinco revistas: Journal of Teaching in Physical Education (editada por Academy of the National Association for Sport and Physical Education \& International Association for Physical Education in Higher Education, Estados Unidos: 1 artículo), Sportis (Universidad de la Coruña, España: 3 artículos), Retos (Federación Española de Asociaciones de Docentes de Educación Física, España: 2 artículos), Voces de la Educación (revista independiente, México: 1 artículo) y Journal Journal of Physical Education and Human Movement (Universidad de Málaga, España: 1 artículo).

En la Figura 1 se presenta el diagrama de flujo del proceso de selección del corpus de estudio para la presente investigación. 


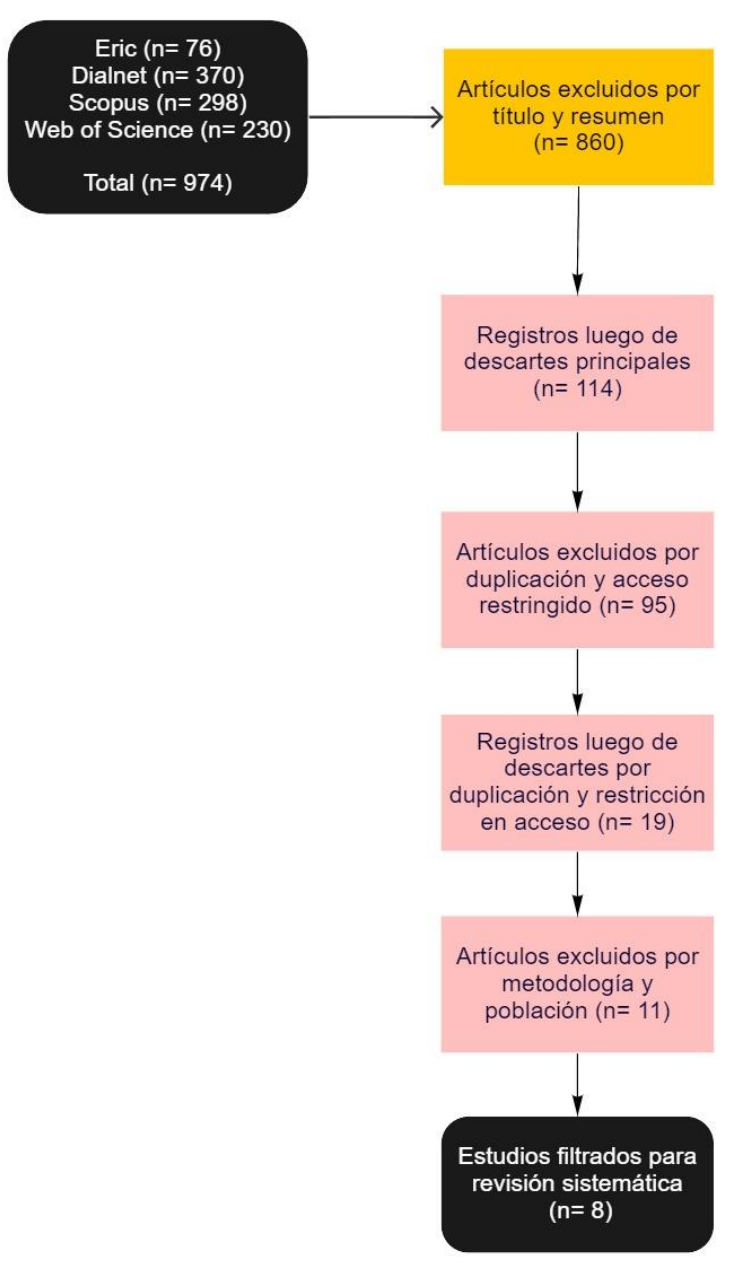

Figura 1. Diagrama de flujo. Fuente: Elaboración propia (2021).

A continuación, se presenta información con respecto a los artículos que fueron seleccionados. Para ello se considera el título, autores, año de publicación, país donde se realizó el estudio, objetivo de investigación, diseño, población, TCM constatado en la clase de EF, instrumentos empleados para la medición, resultados, conclusiones de los estudios (Tabla 1). También se asigna un código para cada artículo (A1-A8), a fin de cotejar los resultados con la lista de verificación de calidad metodológica que se presentará más adelante. 
Tabla 1

Análisis de los artículos seleccionados

\begin{tabular}{|c|c|c|c|c|c|c|c|c|}
\hline Referencia & $\begin{array}{l}\text { Código } \\
\text { País }\end{array}$ & Objetivo & Diseño & Población & TCM & $\begin{array}{c}\text { Instrumento } \\
\text { utilizado }\end{array}$ & Resultados & Conclusiones \\
\hline $\begin{array}{l}\text { Retamal- } \\
\text { Valderrama } \\
\text { et al. (2019) }\end{array}$ & $\begin{array}{l}\text { A2 } \\
\text { Chile }\end{array}$ & $\begin{array}{l}\text { Identificar el nivel } \\
\text { de AF, } \\
\text { comportamiento del } \\
\text { profesor y tiempo } \\
\text { efectivo de las } \\
\text { clases de EF, en una } \\
\text { escuela pública de } \\
\text { Chile. }\end{array}$ & $\begin{array}{l}\text { Observacional, } \\
\text { descriptivo de } \\
\text { corte } \\
\text { transversal. }\end{array}$ & $\begin{array}{l}480 \text { alumnos } \\
\text { ( } 230 \text { niñas y } \\
250 \text { niños) de } \\
\text { primero a } \\
\text { cuarto año } \\
\text { básico. }\end{array}$ & $\begin{array}{l}32 \% \text { del } \\
\text { tiempo de la } \\
\text { clase fue } \\
\text { destinado a } \\
\text { AFMV. }\end{array}$ & $\begin{array}{l}\text { Se utilizó el } \\
\text { instrumento } \\
\text { SOFIT. }\end{array}$ & $\begin{array}{l}\text { El 4,4 \% realiza } \\
\text { intensidad muy activa, el } \\
47,9 \% \text { sentados y el } \\
27,6 \% \text { caminando. El } \\
90,6 \% \text { de la clase, el } \\
\text { profesor no promueve la } \\
\text { práctica de AF, mientras } \\
\text { que el 9,4 \% sí promueve } \\
\text { la AF. }\end{array}$ & $\begin{array}{l}\text { Existe necesidad de } \\
\text { mejorar los tiempos de la } \\
\text { clase de EF, mediante } \\
\text { una mayor participación } \\
\text { del profesor, } \\
\text { transformándose en un } \\
\text { agente promotor y } \\
\text { motivador de la práctica } \\
\text { de AF y hábitos } \\
\text { saludables. }\end{array}$ \\
\hline $\begin{array}{l}\text { Ruiz et al. } \\
\text { (2019) }\end{array}$ & $\begin{array}{l}\text { A3 } \\
\text { España }\end{array}$ & $\begin{array}{l}\text { Analizar y } \\
\text { cuantificar los } \\
\text { diferentes tipos de } \\
\text { tiempos en los que } \\
\text { se fracciona la clase } \\
\text { de EF. La intención } \\
\text { es, por un lado, } \\
\text { concienciar a los } \\
\text { docentes y, por otro, } \\
\text { el desarrollo de } \\
\text { estrategias para que } \\
\text { en estas clases } \\
\text { aumente el TCM. }\end{array}$ & $\begin{array}{l}\text { Observacional } \\
\text { descriptivo, de } \\
\text { corte } \\
\text { transversal. }\end{array}$ & $\begin{array}{l}\text { De } 50 \text { niños, } \\
\text { se seleccionan } \\
\text { sujetos de } \\
\text { manera } \\
\text { aleatoria, } \\
\text { analizando tres } \\
\text { alumnos de } 5 .^{\circ} \\
\text { A (dos niños y } \\
\text { una niña) y } \\
\text { tres de } 5 .^{\circ} \text { B } \\
\text { (un niño y dos } \\
\text { niñas) como } \\
\text { monitores. }\end{array}$ & $\begin{array}{l}\text { El tiempo } \\
\text { real en el que } \\
\text { el niño está } \\
\text { en } \\
\text { movimiento } \\
\text { (TCM) es de } \\
46,67 \% \text {. }\end{array}$ & $\begin{array}{l}\text { Cámara de video } \\
\text { Canon Legria } \\
\text { HFR306. } \\
\text { Programa } \\
\text { Windows Media } \\
\text { para contabilizar } \\
\text { los tiempos. }\end{array}$ & $\begin{array}{l}\text { Teniendo en cuenta la } \\
\text { media de todas las } \\
\text { sesiones, en una clase de } \\
\text { EF de una hora es posible } \\
\text { trabajar } 43,2 \pm 4,2 \text { min, } \\
\text { lo que sería el tiempo útil } \\
\text { de la clase. El tiempo real } \\
\text { en el cual el niño está en } \\
\text { movimiento (TCM) es } \\
\text { solamente de } 21 \text { '. }\end{array}$ & $\begin{array}{l}\text { El TCM en la UD de } \\
\text { vóleibol ha sido superior } \\
\text { a la de bádminton. El } \\
\text { TCM es algo más de } 2 / 4 \text {. } \\
\text { Asimismo, se percibe que } \\
\text { los chicos poseen un } \\
\text { mayor TCM que las } \\
\text { chicas. }\end{array}$ \\
\hline
\end{tabular}




$\begin{array}{lll}\text { Yanci et al. } & \text { A4 } & \text { Analizar el TCM en } \\ \text { (2016) } & \text { España } & \text { función del tipo de } \\ & \text { sesión, curso } \\ & \text { escolar o la hora de } \\ & \text { práctica. } \\ & \text { Comprobar la } \\ & \text { fiabilidad de la } \\ & \text { herramienta de } \\ & \text { observación TiPEF } \\ & \text { para observar, } \\ & \text { codificar y analizar } \\ & \text { el TCM. }\end{array}$

Observacional descriptivo, de corte transversal.

\section{Alumnos}

educación primaria

264 niños y

niñas

(6-9 años).

$\mathrm{EP} 1=98$

$\mathrm{EP} 2=102$

$\mathrm{EP} 3=64$

$\begin{array}{llll}\text { Fernández } & \text { A5 } & \text { Valorar el TCM de } & \text { Observacional } \\ \text { (2019) } & \text { España } & \text { los discentes. } & \text { descriptivo, de } \\ & & & \text { corte } \\ & & \text { transversal. }\end{array}$

Reyes et al. A6

(2020)

Venezuela

Analiza el TCM en

Observacional descriptivo, de clases de EF en tres subsistemas de la

educación venezolana.
El TCM para Herramienta de Los valores obtenidos en todas las observación cuanto a la fiabilidad de

clases

agrupadas

analizadas

fue de 21,4

$\min$.

(rango 10-30

min./sesión)

Solo un

$42,8 \%$ del

tiempo de

clases fue

destinado a

la práctica de

AF. corte transversal.

\section{Se utilizó un \\ ronómetro y \\ hoja de registro. \\ impartidas \\ a primera hora \\ de la mañana, \\ hay un \\ TCM mucho \\ menor que en \\ el resto de las \\ sesiones.}

cronómetro, la

grabación de las

clases y la hoja
20 alumnos

del primer

ciclo de

educación

primaria.

La media del

TCM ha sido

de un

$43,44 \%$

del tiempo

total de

clase, o sea,

19,55

minutos (de

45 minutos).

192 alumnos

entre

enseñanza

inicial, básica

y media. En

enseñanza

primaria

fueron 67 los

participantes.
De las clases Se utilizó un observadas

en ens.

básica, una

tuvo $15 \%$ de

TCM y la

segunda un

TCM de

$41 \%$.

de registro.
El TCM es muy bajo en cualquiera de los tres

niveles en observación, y en enseñanza primaria es dispar, aun así, sigue siendo bajo.
La herramienta de observación TiPEF es un instrumento fiable para medir el tipo de sesiones en función del agrupamiento de los alumnos y el TCM en las sesiones de EF en primaria. En cuanto al TCM, no se observaron diferencias en función del tipo de agrupamiento, pero sí en función del grupo de edad.

El TCM en clase es insuficiente. El horario influye en el incremento del TCM.

El TCM es insuficiente en cualquiera de los niveles en observación. Hay desaprovechamiento en la clase de EF y la didáctica del docente interfiere con el incremento de la $\mathrm{AF}$ en escolares. 


\begin{tabular}{|c|c|c|c|c|c|c|c|c|}
\hline $\begin{array}{l}\text { Galloway et } \\
\text { al. (2019) }\end{array}$ & $\begin{array}{l}\text { A7 } \\
\text { Estados } \\
\text { Unidos }\end{array}$ & $\begin{array}{l}\text { Cuantificar las } \\
\text { diferencias } \\
\text { demográficas de la } \\
\text { AF en la escuela } \\
\text { entre los alumnos } \\
\text { de educación } \\
\text { básica. }\end{array}$ & $\begin{array}{l}\text { Observacional } \\
\text { descriptivo, de } \\
\text { corte } \\
\text { transversal. }\end{array}$ & $\begin{array}{l}148 \text { alumnos, } \\
71 \text { niños y } 77 \\
\text { niñas }\left(4 .^{\circ} \text { año }\right. \\
\text { de enseñanza } \\
\text { primaria). }\end{array}$ & $\begin{array}{l}\text { El TCM } \\
\text { alcanzado } \\
\text { por los niños } \\
\text { varía según } \\
\text { sexo y raza, } \\
\text { y quienes } \\
\text { más alcanzan } \\
\text { a realizar AF } \\
\text { de moderada } \\
\text { a vigorosa } \\
\text { son los niños } \\
\text { no blancos, } \\
\text { con un } \\
31,5 \% \text { del } \\
\text { tiempo de la } \\
\text { clase (60 } \\
\text { minutos). }\end{array}$ & $\begin{array}{l}\text { Se usó un } \\
\text { acelerómetro. }\end{array}$ & $\begin{array}{l}\text { El TCM es muy bajo; el } \\
\text { mayor TCM alcanza } \\
\text { apenas el } 31,5 \% \text { en la } \\
\text { clase de EF; los niños } \\
\text { tienen más AF que las } \\
\text { niñas; los niños no } \\
\text { blancos acumulan más } \\
\text { AF que los niños } \\
\text { blancos. }\end{array}$ & $\begin{array}{l}\text { Hay diferencias } \\
\text { significativas en cuanto a } \\
\text { la cantidad de AF que } \\
\text { realizan los niños, según } \\
\text { sexo y raza. }\end{array}$ \\
\hline $\begin{array}{l}\text { López- } \\
\text { Taveras y } \\
\text { Moya-Mata } \\
\text { (2019). }\end{array}$ & $\begin{array}{l}\text { A8 } \\
\text { España }\end{array}$ & $\begin{array}{l}\text { Analizar el TCM en } \\
\text { función de la sesión } \\
\text { y el curso escolar en } \\
\text { el alumnado de } \\
\text { primer y quinto } \\
\text { curso de Educación } \\
\text { Primaria. }\end{array}$ & $\begin{array}{l}\text { Observacional } \\
\text { descriptivo, de } \\
\text { corte } \\
\text { transversal. }\end{array}$ & $\begin{array}{l}38 \text { estudiantes } \\
\left(18 \text { de } 1 .^{\circ}\right. \\
\text { curso y } 20 \text { del } \\
5 .^{\circ} \text { curso, de } 6 \\
\text { y } 10 \text { años } \\
\text { respectivamen } \\
\text {-te). }\end{array}$ & $\begin{array}{l}\text { El TCM } \\
\text { reportado } \\
\text { corresponde } \\
\text { a una media } \\
\text { en primer } \\
\text { curso de un } \\
28,53 \% \text { y en } \\
\text { quinto curso } \\
\text { de un } \\
27,05 \% \text {. }\end{array}$ & $\begin{array}{l}\text { Se utilizó un } \\
\text { cronómetro, la } \\
\text { grabación de las } \\
\text { clases y una } \\
\text { hoja de registro. }\end{array}$ & $\begin{array}{l}\text { El TCM es muy bajo en } \\
\text { las clases de EF } \\
\text { observadas, siendo } \\
\text { mayor en el primer curso. }\end{array}$ & $\begin{array}{l}\text { Poco más del } 70 \% \text { se } \\
\text { destina a otras } \\
\text { actividades que no } \\
\text { corresponden ni } \\
\text { incentivan la práctica } \\
\text { motriz y AF en clases, } \\
\text { perdiéndose } \\
\text { oportunidades valiosas. }\end{array}$ \\
\hline
\end{tabular}

Fuente: Elaboración propia (2021) 
Tal y como se destaca en la Tabla 1, los ocho artículos seleccionados refieren el TCM o tiempo efectivo en la clase de EF en enseñanza primaria. De ellos, se realizaron cuatro en España, (Fernández, 2019; López-Taveras y Moya-Mata, 2019; Ruiz et al., 2019; Yanci et al., 2016), uno en Estados Unidos (Galloway et al., 2019), uno en México (Flores et al., 2017), uno en Venezuela (Reyes et al., 2020), y uno en Chile (Retamal-Valderrama et al., 2019).

Los ocho artículos seleccionados refieren el TCM y/o tiempo efectivo dentro de la clase de EF en sus objetivos de investigación. Flores et al. (2017), Retamal-Valderrama et al. (2019), y Ruiz et al. (2019) mencionan la medición del tiempo efectivo en la clase de EF. Se presentan en Flores et al. (2017), Galloway et al. (2019), y Retamal-Valderrama et al. (2019) distintas variables de medición (AF de moderada a vigorosa, gasto calórico y comportamiento del profesor) que influyen positivamente en el resultado del tiempo efectivo, como lo destacan Reyes et al. (2020) y López-Taveras y Moya-Mata (2019). En Galloway et al. (2019) se advierte la AF de moderada a vigorosa en relación con el sexo y la raza (niños blancos y niños que no son blancos). La variable 'comportamiento del profesor' en Retamal-Valderrama et al. (2019) proporciona un incremento en los niveles de tiempo efectivo, alcanzando un $32 \%$ en comparación a Flores et al. (2017) con un 18,10\%.

En Fernández (2019) se advierten las variables de hora del día, tipo de actividad realizada y la forma de agrupación de los alumnos para el desarrollo de las actividades. No obstante, es de considerar que este último trabajo no logra sobrepasar el $60 \%$ de los indicadores de calidad metodológica. En Reyes et al. (2020) se consideran variables temporales (horario de clase, hora de inicio y hora de finalización de la clase, tiempo real de la clase, TCM) y variables asociadas a organización de grupos, actividades, materiales y espacio físico, mientras que en LópezTaveras y Moya-Mata (2019) se advierten variables temporales asociadas al tiempo real de AF en la clase, a saber: TCM, tiempo disponible para la práctica, tiempo útil y tiempo de programa.

En cuanto a los diseños de investigación, se evidencia que todos los artículos son de tipo observacional descriptivo, de corte transversal. Por otra parte, si bien la población total en los estudios seleccionados es de 1262 estudiantes de enseñanza primaria, en tres de los artículos no se especifican cantidades diferenciadas de niñas y niños. Entre los artículos que sí especifican el sexo, se tiene que 413 corresponden a niñas y 530 corresponden a niños. Flores et al. (2017), Reyes et al. (2010) y Ruiz et al. (2019) consideran como población alumnos de un nivel educativo igual o mayor a quinto básico, teniendo en cuenta que las poblaciones de estudio en cinco trabajos no superan el nivel educativo de sexto básico, por tratarse del nivel de estudio considerado en la 
revisión de literatura. En Reyes et al. (2020), se advierte que se trabajó con tres niveles de la educación, esto es, educación inicial (o parvularia), educación básica y educación media, por lo que se consideraron para el presente análisis solo aquellos datos correspondientes a la educación básica, lo que fue posible porque los investigadores disgregan los resultados por año, por curso y por nivel educativo, con las cantidades de estudiantes por curso.

Respecto al TCM, este no supera el 46,67 \%. En Reyes et al. (2020) se presenta un TCM de $15 \%$ (el menor de los ocho artículos seleccionados); sin embargo, en Retamal-Valderrama et al. (2019) no se especifica el porcentaje de TCM, sino que este debe calcularse.

En cuanto al nivel de AF, Flores et al. (2017) y Retamal-Valderrama et al. (2019) sostienen que los niveles de AFMV evidenciados en las clases de EF no superan $32 \%$. Ruiz et al. (2019) y Yanci et al. (2016) no miden el nivel de AF, debido a que los instrumentos empleados son observacionales y buscan medir principalmente el tiempo efectivo. Por otra parte, Flores et al. (2017), Galloway et al. (2019) y Retamal-Valderrama et al. (2019) utilizan instrumentos que miden el nivel de AF (acelerómetros y SOFIT) de moderada a vigorosa, además de entregar datos del TCM. Flores et al. (2017) y Retamal-Valderrama et al. (2019) utilizaron la herramienta SOFIT, y en uno de ellos además se incluyó un podómetro (Flores et al., 2017). En el caso de Fernández (2019), se usa solo el cronómetro y hoja de registro, y en Reyes et al. (2020) se emplea el cronómetro, la grabación de las sesiones de clase y la hoja de registro. Ruiz et al. (2019) emplearon una cámara de video para filmar la clase y realizar observaciones posteriores y calcular instancias de tiempo.

Luego de repasados los principales resultados, a continuación, se presenta la lista de verificación de calidad metodológica (Tabla 2). 
Tabla 2

Lista de verificación para el reporte de estudios observacionales descriptivos (MINCIR)

\begin{tabular}{|c|c|c|c|c|c|}
\hline Dominio & Ítem & Pregunta clave & Sí & No & $\begin{array}{c}\text { No } \\
\text { corresponde }\end{array}$ \\
\hline \multirow{7}{*}{ Introducción } & $\begin{array}{l}\text { Problema en } \\
\text { estudio }\end{array}$ & $\begin{array}{l}\text { ¿Desarrolla un enfoque general } \\
\text { del problema en estudio, } \\
\text { de la información científica } \\
\text { disponible y la justificación de la } \\
\text { investigación que se está reportando? }\end{array}$ & $\begin{array}{l}\text { A1, A2, } \\
\text { A3, A4, } \\
\text { A5, A6, } \\
\text { A7, A8 }\end{array}$ & - & - \\
\hline & Objetivos & $\begin{array}{l}\text { ¿Se plantean objetivos claros } \\
\text { y precisos? }\end{array}$ & $\begin{array}{l}\text { A1, A2, } \\
\text { A3, A4, } \\
\text { A5, A6, } \\
\text { A7, A8 }\end{array}$ & - & - \\
\hline & $\begin{array}{l}\text { Diseño del } \\
\text { estudio }\end{array}$ & $\begin{array}{l}\text { ¿Menciona el diseño de estudio } \\
\text { utilizado? }\end{array}$ & $\begin{array}{l}\text { A1, A2, } \\
\text { A3, A4 } \\
\text { A6, A7 } \\
\quad \text { A8 }\end{array}$ & A5 & - \\
\hline & $\begin{array}{l}\text { Lugar de la } \\
\text { investigación }\end{array}$ & $\begin{array}{l}\text { ¿Describe el escenario, lugares } \\
\text { y fechas correspondientes; incluidos } \\
\text { la eventual exposición, seguimiento } \\
\text { y recopilación de datos? }\end{array}$ & $\begin{array}{l}\mathrm{A} 1, \mathrm{~A} 2 \\
\mathrm{~A} 5, \mathrm{~A} 6 \\
\mathrm{~A} 7, \mathrm{~A} 8\end{array}$ & $\begin{array}{l}\text { A3 } \\
\text { A4 }\end{array}$ & - \\
\hline & Participantes & $\begin{array}{l}\text { ¿Indica el número de sujetos } \\
\text { estudiados o el tamaño de la muestra } \\
\text { utilizada? }\end{array}$ & $\begin{array}{l}\text { A1, A2, } \\
\text { A3, A4, } \\
\text { A5, A6, } \\
\text { A7, A8 }\end{array}$ & - & - \\
\hline & $\begin{array}{l}\text { Criterios de } \\
\text { inclusión }\end{array}$ & $\begin{array}{l}\text { ¿Señala los criterios de inclusión } \\
\text { de la población estudiada? }\end{array}$ & A7 & - & $\begin{array}{c}\mathrm{A} 1, \mathrm{~A} 2, \mathrm{~A} 3 \\
\mathrm{~A} 4, \mathrm{~A} 5, \mathrm{~A} 6 \\
\mathrm{~A} 8\end{array}$ \\
\hline & $\begin{array}{l}\text { Criterios de } \\
\text { exclusión }\end{array}$ & $\begin{array}{l}\text { ¿Cita los criterios de exclusión } \\
\text { de la población estudiada? }\end{array}$ & - & - & $\begin{array}{l}\mathrm{A} 1, \mathrm{~A} 2, \mathrm{~A} 3 \\
\mathrm{~A} 4, \mathrm{~A} 5, \mathrm{~A} 6 \\
\mathrm{~A} 7, \mathrm{~A} 8\end{array}$ \\
\hline \multirow[b]{2}{*}{ Metodología } & Muestreo & $\begin{array}{l}\text { ¿Indica el tipo de muestreo utilizado? } \\
\text { (cuando corresponda) }\end{array}$ & $\begin{array}{l}\text { A1, A2, } \\
\text { A3, A4, } \\
\text { A5, A6, } \\
\text { A7, A8 }\end{array}$ & - & - \\
\hline & Variables & $\begin{array}{l}\text { ¿Define claramente las variables } \\
\text { estudiadas? Idealmente la variable } \\
\text { principal o de resultado y “otras } \\
\text { variables de interés”. Si corresponde, } \\
\text { señalar quién, cómo, con qué } \\
\text { y cuándo midió. }\end{array}$ & $\begin{array}{l}\text { A1, A2, } \\
\text { A3, A4, } \\
\text { A5, A6, } \\
\text { A7, A8 }\end{array}$ & - & - \\
\hline
\end{tabular}




\begin{tabular}{|c|c|c|c|c|c|}
\hline & Seguimiento & $\begin{array}{l}\text { ¿Menciona el tiempo de observación } \\
\text { o de seguimiento de los sujetos } \\
\text { en estudio? (según corresponda) }\end{array}$ & $\begin{array}{l}\text { A1, A2, } \\
\text { A3, A4, } \\
\text { A5, A6, } \\
\text { A7, A8 }\end{array}$ & - & - \\
\hline & Estadísticas & $\begin{array}{l}\text { ¿Señala las herramientas estadísticas } \\
\text { utilizadas? Referirse a las estadísticas } \\
\text { descriptivas y analíticas que } \\
\text { se emplearon (según corresponda). }\end{array}$ & $\begin{array}{l}\text { A1, A2, } \\
\text { A3, A4, } \\
\text { A5, A6, } \\
\text { A7, A8 }\end{array}$ & - & - \\
\hline & $\begin{array}{l}\text { Principios } \\
\text { éticos }\end{array}$ & $\begin{array}{l}\text { ¿Indica los principios éticos } \\
\text { involucrados? }\end{array}$ & $\begin{array}{l}\text { A1, A2 } \\
\text { A3, A4 } \\
\text { A6, A7 } \\
\quad \text { A8 }\end{array}$ & A5 & - \\
\hline & Participantes & $\begin{array}{l}\text { ¿Describe de forma general } \\
\text { la muestra estudiada? }\end{array}$ & $\begin{array}{l}\text { A1, A2 } \\
\text { A3, A4 } \\
\text { A5, A6, } \\
\text { A7, A8 }\end{array}$ & - & - \\
\hline \multirow[b]{2}{*}{ Resultados } & $\begin{array}{l}\text { Análisis de } \\
\text { grupos y } \\
\text { subgrupos }\end{array}$ & $\begin{array}{l}\text { ¿Aplica estadística analítica } \\
\text { y comparación de grupos y } \\
\text { subgrupos? (cuando corresponda) }\end{array}$ & $\begin{array}{l}\text { A1, A2 } \\
\text { A3, A4 } \\
\text { A5, A6, } \\
\text { A7, A8 }\end{array}$ & - & - \\
\hline & Otros análisis & $\begin{array}{l}\text { ¿Emplea otro tipo de análisis } \\
\text { estadístico? Por ejemplo: análisis } \\
\text { de supervivencia, ajustes por factores } \\
\text { de confusión y su precisión } \\
\text { estimando los intervalos de confianza } \\
\text { del } 95 \% \text { (según corresponda) }\end{array}$ & $\mathrm{A} 4, \mathrm{~A} 7$ & $\begin{array}{l}\text { A1, } \\
\text { A2, } \\
\text { A3, } \\
\text { A5 } \\
\text { A6, } \\
\text { A8 }\end{array}$ & - \\
\hline \multirow{4}{*}{ Discusión } & $\begin{array}{l}\text { Novedad de la } \\
\text { propuesta }\end{array}$ & $\begin{array}{l}\text { ¿Discute acerca de los aspectos } \\
\text { novedosos del estudio que } \\
\text { se presenta? }\end{array}$ & $\begin{array}{l}\text { A1, A2 } \\
\text { A3, A4 } \\
\text { A6, A7 } \\
\quad \text { A8 }\end{array}$ & A5 & - \\
\hline & $\begin{array}{l}\text { Comentarios } \\
\text { respecto de los } \\
\text { resultados }\end{array}$ & $\begin{array}{l}\text { ¿Comenta e interpreta los resultados } \\
\text { obtenidos en relación } \\
\text { al conocimiento existente } \\
\text { y resultados de estudios similares? }\end{array}$ & $\begin{array}{l}\mathrm{A} 1, \mathrm{~A} 2 \\
\mathrm{~A} 3, \mathrm{~A} 4 \\
\mathrm{~A} 6, \mathrm{~A} 7 \\
\quad \mathrm{~A} 8\end{array}$ & A5 & - \\
\hline & $\begin{array}{l}\text { Limitaciones } \\
\text { del estudio }\end{array}$ & $\begin{array}{l}\text { ¿Expone las limitaciones del estudio } \\
\text { y los potenciales sesgos existentes } \\
\text { en él? }\end{array}$ & $\begin{array}{c}\text { A2, A4, } \\
\text { A6, A7 }\end{array}$ & $\begin{array}{l}\text { A1 } \\
\text { A3 } \\
\text { A5 } \\
\text { A8 }\end{array}$ & - \\
\hline & Conclusión(es) & $\begin{array}{l}\text { ¿Plantea una conclusión? Solo para } \\
\text { aquellos estudios en los que se pueda } \\
\text { plantear. }\end{array}$ & $\begin{array}{l}\text { A1, A2, } \\
\text { A3, A4 } \\
\text { A5, A6 } \\
\text { A7, A8 }\end{array}$ & - & - \\
\hline
\end{tabular}

Fuente: Elaboración propia (2021) 
Para la verificación de calidad metodológica de los estudios seleccionados se usó la lista de reporte MINCIR, instrumento validado para estudios observacionales descriptivos (Manterola y Astudillo, 2013). Según esto: A3 y A4 no dan cuenta de los lugares en los que se hizo la investigación en cuestión; A1, A3 y A5 carecen de información referida a limitaciones del estudio y/o posible sesgo. Todos los artículos tienen análisis estadístico; A4 y A7 incorporan intervalos de confianza del $95 \%$, mientras que A1, A2, A3, A5, A6 no ofrecen información al respecto. Debido al tipo de muestreo, ninguno de los artículos declara criterios de inclusión y exclusión; A5 no declara aspectos éticos involucrados. Así, de 19 criterios, hay dos de ellos (criterios de inclusión y exclusión) que no corresponden a ninguno de los artículos; A1 cumple 15 criterios (78,95 \%), A2 cumple $16(84,21 \%)$, A3 cumple 14 (73,68 \%), A4 cumple con 16 (84,21\%), A5 cumple solo $11(57,89 \%)$, A6 cumple 16 criterios (84,21\%), A7 cumple con 18 (94,73\%) y A8 cumple 15 criterios (78,95\%). Los artículos A2, A4, A6 y A7 están sobre el $80 \%$ en el cumplimiento de los dominios estructurales y metodológicos considerados, A1 y A8 se quedan muy cerca del $80 \%$, A3 supera el $70 \%$ y A5 no alcanza a superar el $60 \%$ de los criterios.

\section{Discusión}

Los resultados advierten poca investigación en América Latina (AL) y Chile sobre TCM. Además, se evidencia poca investigación sobre TCM en enseñanza primaria, más aún en AL (tres de ocho estudios son en AL) en razón de tres elementos necesarios: en primer lugar, la cantidad de tiempo que según organizaciones como la OMS (Bull et al., 2021) se sugieren para que niños y niñas realicen AF; en segundo lugar, y como sostiene la misma OMS, que para muchos niños y adolescentes la clase de EF es la única instancia que tienen para realizar AF, y en último término, el tiempo aprovechable en la clase de EF que incide en el aprendizaje motor, tal y como lo sostienen, en sus respectivas investigaciones, Olmedo (2000) y Siedentop (1998).

Los datos extraídos de las evidencias muestran que el TCM es bajo en la clase de EF (el porcentaje de TCM más alto es de 46,67\%), lo que además se confirma en Ministerio de Educación (2020) y en Torres (2011). Este último declara: "Es significativamente insuficiente el tiempo destinado al desarrollo por parte de los alumnos de actividades motrices dirigidas al cumplimiento de los objetivos propuestos para la clase”. En Salazar et al. (2017), los estudiantes pasan un $63 \%$ de tiempo de la clase de EF, en posición de pie, esperando turno para ejecutar, escuchando indicaciones del profesor o restándose de participar. En López-Taveras y Moya- 
Mata (2019) se advierte que poco más del $70 \%$ de la clase se pierde sin posibilidades de generar motivación para la práctica motriz, y en Fernández (2019), quien hizo un estudio en educación primaria, "la media del tiempo de compromiso motor ha sido de un 43,44\% del tiempo total de clase" (p. 41), constatando que la gestión de tiempo se asocia con la disminución de posibilidades para el aprendizaje y el desarrollo motor.

En el caso de López-Taveras y Moya-Mata (2019), dado que se trabajó con dos cursos (1. y 5. ${ }^{\circ}$ de educación primaria), se advierte que en el primer curso el TCM es mayor al curso de 5. , lo que es compatible con los resultados de Guthold et al. (2019) al señalar que, a medida que niños y niñas van creciendo, la adherencia a la AF disminuye. Esta situación se ha visto patente en Chile tras los resultados entregados por el Ministerio de Educación (2020) a raíz de los porcentajes dedicados a la AF por niños y niñas en los distintos niveles educacionales $(8,5 \%$ del tiempo escolar para $\mathrm{AF}$ entre $1 .^{\circ}$ y $4 .^{\circ}$ básico y $4,25 \%$ entre $5 .^{\circ}$ y $6 .^{\circ}$ básico).

Uno solo de los estudios seleccionados se hizo en Chile (Retamal-Valderrama et al., 2019), y en este se determinó que el TCM fue de $32 \%$, estando asociado a la AFMV. Estos resultados son relevantes, dado que Chile se ubicó en el penúltimo lugar entre 49 países de la Organización para la Cooperación y el Desarrollo Económico (OCDE; Aubert et al., 2018) en lo que se refiere a la prevalencia de AF en niños y jóvenes. De acuerdo con este mismo estudio, el $80 \%$ de los niños en edades escolares es inactivo en cuanto a la AF se refiere. Esto preocupa ya que "para muchos niños, especialmente los procedentes de entornos menos favorecidos, la educación física representa las únicas sesiones regulares de actividad física" (Organización de las Naciones Unidas para la Educación, la Ciencia y la Cultura, 2015, p. 14). Así, considerando que la clase de EF está siendo poco aprovechada, mayores podrían ser las secuelas en términos de riesgos de la salud asociados a la falta de AF.

Estos datos se replican a su vez en Chile en tanto la prevalencia de inactividad física en niños varones entre 11 y 17 años para 2016 estaba en el orden de 84,2 \% y, en niñas, en un 91,2\% (Guthold et al., 2019). Nótese que, según datos de la "Encuesta Nacional de Hábitos de Actividad Física y Deporte 2018 en población de 18 años y más”, realizada por el Ministerio del Deporte (2018), el 81,3 \% de las personas estudiadas son inactivas físicamente. Esto es importante porque, según el Reporte de Notas de Actividad Física (Universidad de la Frontera \& Active Healthy Kids Global Alliance, 2018), a medida que los niños y las niñas van creciendo reducen la realización de AF. Este dato se valida y constata en la investigación de Reyes et al. (2020) a nivel de TCM en 
enseñanza media, pero que también puede constatarse en Sallis et al. (1993) y Weinberg \& Gould (1995).

Aunque el interés por realizar algún tipo de AF ha crecido entre 2006 y 2018 (Ministerio del Deporte, 2018), y a pesar de que la percepción respecto a la importancia de la AF y el deporte dentro de las prioridades de Chile ha ido aumentando progresivamente (Ministerio del Deporte, 2018), lo cierto es que existe un decrecimiento constante desde 2006 en la práctica concreta de AF. Es decir, no se está revirtiendo la situación, sino que, al contrario, se agudiza. El interés por realizar AF y el reconocimiento de la importancia de la AF en la salud no está generando una mayor adopción de estilos de vida saludables en lo referente a la práctica de AF de forma regular. El bajo TCM en la clase podría estar agudizando la prevalencia de inactividad física. Se trata de un problema que no se acaba en la infancia, sino que se incrementa en edades posteriores (adolescencia, juventud y vida adulta). De hecho, Camacho-Miñano et al. (2013) sostienen que la transición entre educación básica y educación media es el momento en el que los niños comienzan a abandonar la práctica de AF. Esta es la etapa en la que se consolidan hábitos de práctica que se perpetúan en la edad adulta (Gordon-Larsen et al., 2004; Telama, 2009), de allí que tales resultados sean relevantes y, a la vez, reveladores. Es notable que los niveles de AFMV en las clases de EF no superen el $32 \%$ (Flores et al., 2017; Retamal-Valderrama et al., 2019), un dato que verifica bajos niveles de AF constados ya en otras investigaciones en Chile (Mayorga-Vera et al., 2020), en la que ninguno de los participantes (156) alcanzó siquiera el $50 \%$ del tiempo requerido en la clase de EF (Ministerio del Deporte et al., 2017).

En relación con los instrumentos empleados, hay variedad en tanto se emplean acelerómetros, podómetros, cronómetros, hojas de registro y grabación de clases, siendo el más recurrente el acelerómetro. La selección del instrumento es un dato de relevancia, dado que, mientras más preciso sea el instrumento, mayor será la calidad de la información entregada (Pérez, 2018). En una revisión sistemática realizada en función de la acelerometría, Calahorro et al. (2015) concluyen que, si bien es cierto no existe uniformidad en los criterios técnicos empleados en el uso de acelerómetros, estos se presentan como herramientas sencillas y prácticas para cuantificar y conocer de forma más detallada los niveles y patrones de AF con sujetos en edad escolar. Por supuesto, esto no implica el desconocimiento del uso del cronómetro para la medición, no obstante, con este último se requiere que el observador posea criterios muy claros y protocolarmente definidos, al tiempo que ejecute con precisión y con el acompañamiento de un segundo observador. De hecho, se advierte en Rodríguez y Terrados (2006) una caracterización de instrumentos para 
medición de actividad física, declarando ventajas y desventajas de diversos instrumentos, entre ellos los ya mencionados: podómetro, acelerómetro y cronómetro. Por último, Jurado-Castro et al. (2019), además de destacar el podómetro, el acelerómetro y pulseras, suponen el uso de técnicas como el agua doblemente marcada, la calorimetría y la observación directa (cronómetros), entre otros.

\begin{tabular}{|c|c|c|c|c|c|c|}
\hline Metodología & Validación & Objetividad & Reproducibilidad & $\begin{array}{l}\text { Asequibilidad } \\
\text { económica }\end{array}$ & $\begin{array}{c}\text { Facilidad de } \\
\text { uso }\end{array}$ & Dimensiones de la AF \\
\hline Acelerometría & $\checkmark \checkmark \checkmark \checkmark$ & $\checkmark \checkmark \checkmark \checkmark$ & $\sqrt{ } \sqrt{ }$ & $\sqrt{ }$ & $\sqrt{ } \sqrt{ }$ & $\sqrt{ } \sqrt{ }$ \\
\hline $\begin{array}{l}\text { Agua doblemente } \\
\text { marcada }\end{array}$ & $\sqrt{ } \checkmark \checkmark \checkmark$ & $\checkmark \checkmark \checkmark V$ & $\checkmark$ & $\checkmark$ & $\checkmark$ & $\checkmark$ \\
\hline Calorimetría & $\checkmark \checkmark \checkmark \checkmark$ & $\checkmark \checkmark \checkmark \checkmark$ & $\checkmark$ & $\checkmark$ & $\checkmark$ & $\checkmark$ \\
\hline Cuestionarios & $\checkmark \sqrt{ }$ & $\checkmark$ & $\checkmark \sqrt{ } \sqrt{ }$ & $\checkmark \vee V \checkmark$ & $\checkmark \vee \vee V$ & $\sqrt{ }$ \\
\hline $\begin{array}{l}\text { Diarios de } \\
\text { actividades }\end{array}$ & $\checkmark V$ & $\checkmark$ & $\checkmark \checkmark \vee \checkmark$ & $\checkmark \vee \vee V$ & $\checkmark \checkmark \checkmark V$ & $\checkmark \checkmark \checkmark$ \\
\hline Observación directa & $\checkmark v$ & $\checkmark V$ & $\checkmark \vee \vee V$ & $\checkmark$ & $\checkmark \checkmark V$ & $\checkmark \checkmark \checkmark V$ \\
\hline Podometría & $\sqrt{ } V$ & $\checkmark V V$ & $\sqrt{ } \checkmark V$ & $\checkmark \vee \vee V$ & $\checkmark \vee \vee \checkmark$ & $\checkmark$ \\
\hline $\begin{array}{l}\text { Pulseras de } \\
\text { actividad }\end{array}$ & $\sqrt{ }$ & $\sqrt{ } V$ & $\sqrt{ }$ & 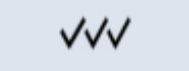 & $\checkmark \vee V \checkmark$ & $\sqrt{ } \checkmark \checkmark$ \\
\hline
\end{tabular}

Figura 2. Valoración de diferentes herramientas. Fuente: Jurado-Castro et al. (2019).

Vale destacar que, en Fernández (2019), Galloway et al. (2019), y en Ruiz et al. (2019), los niños logran más TCM que las niñas, pero este dato se contrasta con los datos que ofrece Siedentop (1998), quien sostiene que las niñas alcanzan un porcentaje de TCM similar a los niños en la clase de EF. El caso del estudio de Galloway et al. (2019) es el único que consideró diferencias en razón del TCM alcanzado por niños blancos y no blancos, siendo estos últimos quienes alcanzaron mayor cantidad de AF de moderada a vigorosa en la semana de estudio. El contenido desarrollado en clases podría favorecer el incremento del TCM. Así, en Yanci et al. (2016) se advierte que el trabajo con la disciplina de vóleibol presenta mayor TCM en comparación con el bádminton, por ejemplo. En este sentido, Siedentop (1998) afirma que el TCM sí varía de acuerdo con las actividades que se realizan en la clase de EF, aunque plantea que disciplinas deportivas 
individuales y colectivas fomentan menor TCM en comparación con actividades como la danza y acondicionamiento físico, destacando por ejemplo la disyuntiva entre actividades que son netamente de oposición y actividades que no lo son, como por ejemplo actividades colaborativas (sin oposición) y actividades de libre realización.

\section{Conclusiones}

Al considerar las preguntas que orientan esta revisión de literatura, habría que afirmar que las evidencias que se encuentran con respecto al TCM en la clase de EF en enseñanza primaria son pocas. Se hace necesario investigar con mayor asiduidad sobre TCM en Chile y AL en los contextos señalados, a partir de intervenciones que no solo midan y cuantifiquen TCM y niveles de AF, sino que impliquen acciones concretas y metodologías que incrementen el TCM y los niveles de AF en la clase de EF.

La evidencia muestra que el TCM en los alumnos de enseñanza primaria en la clase de EF es bajo y no impacta positivamente en el cumplimiento de las recomendaciones mínimas de la OMS y otras instituciones en cuanto a la AF en la semana para niños y niñas en edades escolares, más aún cuando Chile presenta datos de inactividad física alarmantes en la población infantojuvenil. Dado que en los niños varones se evidencia un mayor TCM en la clase de EF, se hace previsible el desarrollo de actividades que favorezcan la inclusión y potencien la participación de las niñas. Además, actividades de tipo colaborativo y actividades de libre realización son proclives a producir un incremento en el TCM en la clase de EF.

Por otra parte, el TCM observado es considerado insuficiente, y en los trabajos seleccionados para el estudio se endosa la responsabilidad principalmente al profesor por su poca participación, ya sea por no incentivar a los alumnos a realizar las actividades determinadas en la clase de EF o por destinar mucho tiempo a las indicaciones y organización de materiales. Así las cosas, la gestión del tiempo en la clase de EF se convierte en un tema relevante, habida cuenta las políticas públicas educativas y las reformas que en Chile se están ejecutando, siendo la de mayor contundencia la que implica la desestimación de la asignatura de EF como asignatura obligatoria en los años finales de la enseñanza media, esto es, $3 .^{\circ}$ y $4 .^{\circ}$ medio.

Finalmente, las evidencias muestran que el TCM se ve incrementado según la unidad didáctica que se desarrolle y aumenta en edades tempranas (6 a 7 años) en comparación a edades avanzadas (11 a 12 años), siendo mayor en los niños y disminuyendo significativamente en las 
niñas, lo que permite inferir que las prácticas escolares equitativas son necesarias a fin de generar equilibrios en la atención escolar, además de incrementar el TCM en las clases a partir de actividades y metodologías en unidades didácticas que potencien mayor tiempo y volumen de AF.

\section{Posibles líneas de investigación}

A raíz de los resultados obtenidos, se advierten intereses de investigación en función del TCM medido a partir de mecanismos e instrumentos como la podometría, la acelerometría, entre otros. Además, se genera interés en función de la investigación sobre el TCM en enseñanza media y los valores cuantitativos resultantes en la transición entre la enseñanza primaria y la enseñanza media, considerando variables como la metodología de enseñanza en las clases, el TCM en función del sexo, las edades, unidades didácticas desarrolladas en la clase. Incluso puede considerarse el TCM en niños, niñas y adolescentes en situación de discapacidad, entre otros aspectos de interés.

\section{Limitaciones del estudio}

La principal limitación de esta revisión reside en la poca cantidad de estudios filtrados, y ello guarda relación directa con dos elementos: a) la calidad metodológica de los estudios y b) poca investigación en el contexto del TCM, especialmente en América Latina. Una segunda limitación se constata en que esta revisión de literatura no trabajó de forma específica alguna variable, sino que fue advirtiendo en los estudios filtrados cuáles fueron las variables consideradas en cada uno. Pero no fue propósito de esta revisión dedicarse a alguna variable en específico.

\section{Referencias}

Aguilar-Farías, N., Martino-Fuentealba, P., Infante-Grandon, G., \& Cortinez-O’Ryan, A. (2017). Inactividad física en Chile: debemos responder al llamado global. Revista Médica de Chile, 145(12), 1631-1632. https://doi.org/10.4067/s0034-98872017001201631

Aubert, S., Barnes, J. D., Abdeta, C., Abi Nader, P., Adeniyi, A. F., Aguilar-Farías, N., Andrade T., D. S., Bhawra, J., Brazo-Sayavera, J., Cardon, G., Chang, C. K., Delisle N., C., Demetriou, Y., Draper, C. E., Edwards, L., Emeljanovas, A., Gába, A., Galaviz, K. I., González, S. A. ..., \& Tremblay, M. S. (2018). Global matrix 3.0 physical activity report card grades for children and youth: results and analysis from 49 countries. Journal of Physical Activity and Health, 15(2), 251-273. https://doi.org/10.1123/jpah.2018-0472

Bevans, K., Leslie-Anne F., Sánchez, B., Riley, A., \& Forrest, C. (2010). Physical education resources, class management, and student physical activity levels: a structure-process-outcome approach to evaluating 
physical education effectiveness. Journal of School Health, 80(12), 573-580. https://doi.org/10.1111/j.17461561.2010.00544.x

Blanco, M., Veiga, O. L., Sepúlveda, A. R., Izquierdo-Gómez, R., Román, F. J., López, S., \& Rojo, M. (2020). Ambiente familiar, actividad física y sedentarismo en preadolescentes con obesidad infantil: estudio $\begin{array}{lllll}\text { ANOBAS de } & \text { casos-controles. } & \text { Atención }\end{array}$ https://doi.org/10.1016/j.aprim.2018.05.013

Bull, F. C., Al-Ansari, S. S., Biddle, S., Borodulin, K., Buman, M. P., Cardon, G., Carty, C., Chaput, J-P., Chastin, S., Chou, R., Dempsey, P. C., DiPietro, L., Ekelund, U., Firth, J., Friedenreich, C. M., García, L., Gichu, M., Jago, R., Katzmarzyk, P., \& ... Willumsen, J. F. (2020). World Health Organization 2020 guidelines on physical activity and sedentary behaviour. British Journal of Sports Medicine, 54(24), 1451-1462. https://doi.org/10.1136/bjsports-2020-102955

Calahorro, F., Torres-Luque, G., López-Fernández, I., Santos-Lozano, A., Garatachea, N., \& Álvarez C., E. (2015). Actividad física y acelerometría; orientaciones metodológicas, recomendaciones y patrones. Nutrición Hospitalaria, 31(1), 115-128. https://doi.org/10.3305/nh.2015.31.1.7450

Camacho-Miñano, M. J., Fernández G., E., Ramírez R., E., \& Blández A., J. (2013). La educación física escolar en la promoción de la actividad física orientada a la salud en la adolescencia: una revisión sistemática de programas de intervención. Revista Complutense de Educación, 24(1), 9-26. https://doi.org/10.5209/rev_RCED.2013.v24.n1.41189

Carrillo B., S. (2020). El ejercicio físico, la actividad física. ¿Cómo continuarlo en tiempo de pandemia? Revista Costarricense de Cardiología, 22, 1-3. https://www.scielo.sa.cr/pdf/rcc/v22s1/1409-4142-rcc-22-s1-27.pdf

Carroll, J., \& Spearrit, D. (1967). A sutdy of a "Model for school learning". Harvard University, Cambridge. Center for Research and Development in Educational Differences. https://files.eric.ed.gov/fulltext/ED045477.pdf

Cavill, N., Biddle, S., \& Sallis, J. F. (2016). Health enhancing physical activity for young people: statement of the United Kingdom expert consensus conference. Pediatric Exercise Science, 13(1), 12-25. https://doi.org/10.1123/pes.13.1.12

Celis-Morales, C., Salas-Bravo, C., Yáñez, A., \& Castillo, M. (2020). Inactividad física y sedentarismo. La otra cara de los efectos secundarios de la pandemia de COVID-19. Revista Médica de Chile, 148(6), 885-886. https://doi.org/10.4067/S0034-98872020000600885

Chávez, M. E., Margarita S., C., Hoyos R., G., Bautista J., A., González L., D. \& Ogarrio P., C. E. (2018). Actividad física y enfermedades crónicas no transmisibles de estudiantes mexicanos en función del género. Retos, 33, 169-174. https://doi.org/10.47197/retos.v0i33.55354

Coe, D., Pivarnik, J., Womack, C., Reeves, M., \& Malina, R. (2006). Effect of physical education and activity levels on academic achievement in children. Medicine and Science in Sports and Exercise, 38(8), 1515-1519. https://doi.org/10.1249/01.mss.0000227537.13175.1b

Consejo Nacional de Educación de Chile. (2019). Acuerdo $N^{\circ}$ 057/2019. Resolución exenta $N^{\circ} 159$. https://www.cned.cl/sites/default/files/acuerdo_057_2019_res_159_2019_plan.pdf

Díaz-Martínez, X., Petermann, F., Leiva, A. M., Garrido-Méndez, A., Salas-Bravo, C., Martínez, M. A., Labraña, A. M., Duran, E., Valdivia-Moral, P., Zagalaz, M. L., Poblete-Valderrama, F., Alvarez, C., \& Celis-Morales, C. 
(2018). No cumplir con las recomendaciones de actividad física se asocia a mayores niveles de obesidad, diabetes, hipertensión y síndrome metabólico en población chilena. Revista Médica de Chile, 146(5), 585595. https://doi.org/10.4067/s0034-98872018000500585

Fernández, G., N. (2019). Tiempo de compromiso motor en educación primaria. Journal of Physical Education and Human Movement, 1(2), 37-44. https://doi.org/10.24310/JPEHMjpehm.v1i2.6686

Ferreira, I., Urrútia, G., \& Alonso-Coello, P. (2011). Revisiones sistemáticas y metaanálisis: bases conceptuales e $\begin{array}{lllll}\text { interpretación. Revista } & \text { Española de }\end{array}$ https://doi.org/10.1016/j.recesp.2011.03.029

Flores, P. J., Salazar, C. M., Gómez F., J. A., Barreto V., Y., Valdovinos G., O., Vicente R., J. U., \& Del Río Valdivia, J. E. (2017). Medición del tiempo efectivo de la clase de educación física y su impacto en el gasto calórico en escolares de nivel primaria del municipio de Colima, México. Sportis, Revista Técnico-Científica del Deporte Escolar, Educación Física y $\quad$ Psicomotricidad, $\quad 3(1), \quad 34-49$. https://doi.org/10.17979/sportis.2017.3.1.1766

Galloway, R., Booker, R., \& Owens, S. (2019). Factors leading to discrepancies in accumulated physical activity during school hours in elementary school students. Journal of Teaching in Physical Education, 38(4), 338346. https://doi.org/10.1123/jtpe.2018-0232

García, M., Peiró, R., \& López, M. (2012). Sedentary lifestyle of adolescent andalusian. Journal of Sport and Health Research, 4(1), 67-82. http://www.journalshr.com/papers/Vol\%204_N\%201/V04_1_7.pdf

González, J., y Portolés, A. (2014). Actividad física extraescolar: relaciones con la motivación educativa, rendimiento académico y conductas asociadas a la salud extracurricular. Revista Iberoamericana de Psicología del Ejercicio y el Deporte, 9(1), 51-65. https://www.redalyc.org/articulo.oa?id=311130199005

Gordon-Larsen, P., Nelson, M. C., \& Popkin, B. M. (2004). Longitudinal physical activity and sedentary behavior trends: adolescence to adulthood. American Journal of Preventive Medicine, 31(4), 353-353. https://doi.org/10.1016/j.amepre.2004.07.006

Gracia, E., \& Ruiz T., G. (2017). Análisis del tiempo de compromiso motor en Educación Física. EmásF: Revista Digital de Educación Física, 45(45), 31-51. http://tauja.ujaen.es/handle/10953.1/1018

Guthold, R., Stevens, G., Riley, L., \& Bull, F. (2019). Global trends in insufficient physical activity among adolescents: a pooled analysis of 298 population-based surveys with 1.6 million participants. The Lancet. Child \& Adolescent Health, 19, 1-13. https://doi.org/10.1016/S2352-4642(19)30323-2

Junta Nacional de Auxilio Escolar y Becas. (2018). Informe mapa nutricional 2017: situación nutricional de los preescolares y escolares de establecimientos municipalizados y particulares subvencionados del país. https://www.junaeb.cl/wp-content/uploads/2013/03/Informe-Mapa-Nutricional-2017-FINAL.pdf

Jurado-Castro, J. M., Llorente-Cantarero, F. J., \& Gil-Campos, M. (2019). Evaluación de la actividad física en niños. $\begin{array}{llll}\text { Acta Pediátrica } & \text { Española, } & \text { 94-99. }\end{array}$ https://www.actapediatrica.com/index.php/secciones/revision/1605-evaluacion-de-la-actividad-fisica-enninos\#.YLN7-qhKjIU 
Preocupante realidad: Sólo 14 minutos de ejercicio activo en clases de Educación Física. (27 de octubre de 2014). Chile Vive Sano. http://www.chilevivesano.cl/noticias/preocupante-realidad-solo-14-minutos-de-ejercicioactivo-en-clases-de-educacion-fisica

Leiva, A., Petermann-Rocha, F., Martínez-Sanguinetti, M. A., Troncoso-Pantoja, C., Concha, Y., Garrido-Méndez, A., Díaz-Martínez, X., Lanuza-Rilling, F., Ulloa, N., Martorell, M., Álvarez, C., \& Celis-Morales, C. (2018). Asociación de un índice de estilos de vida saludable con factores de riesgo cardiovascular en población chilena. Revista Médica de Chile, 146(12), 1405-1414. https://doi.org/10.4067/s0034-98872018001201405

López de D’Amico, R. (2019). Educación física de calidad: ¿de dónde surge este planteamiento? Revista Caribeña de Investigación Educativa, 3(2), 33-45. https://doi.org/10.32541/recie.2019.v3i2.pp33-45

López-Taveras, A., \& Moya-Mata, I. (2019). ¿Es esto educación física?: el tiempo de compromiso motor en educación primaria. Sportis, Revista Técnico-Científica del Deporte Escolar, Educación Física y Psicomotricidad, 5(3), 373-391. https://doi.org/10.17979/sportis.2019.5.3.5238

Manterola, C., \& Astudillo, P. (2013). Lista de verificación para el reporte de estudios observacionales descriptivos. Iniciativa MINCIR. International Journal of Morphology, 31(1), 115-120. https://doi.org/10.4067/S071795022013000100018

Marques, A., Gómez, F., Martins, J., Catunda, R., \& Sarmento, H. (2017). Association between physical education, school-based physical activity, and academic performance. A systematic review. Retos, 31, 316-320. https://recyt.fecyt.es/index.php/retos/article/view/53509/32245

Márquez Arabia, J. J. (2020). Inactividad física, ejercicio y pandemia COVID-19. Revista de Educación Física, 9(2), 43-56. https://recyt.fecyt.es/index.php/retos/article/view/53509/32245

Márquez, R., Rodríguez, J., \& De Abajo, S. (2006). Sedentarismo y salud: efectos beneficiosos de la actividad física. Apunts Educación Física y Deportes, 83, 12-24. https://core.ac.uk/download/pdf/41584471.pdf

Martins, J., Torres, B., Cardoso, J., Costa, A., \& Honório, S. (2016). Influence of sociological aspects on the level of physical activity in physical education students. Journal of Human Sport and Exercise, 10(3), 815-826. https://doi.org/10.14198/jhse.2015.103.07

Mayorga-Vera, D., Parra S., M., \& Viciana, J. (2020). Niveles objetivos de actividad física durante las clases de Educación Física en estudiantes chilenos usando acelerometría. Retos, 37, 123-128. https://doi.org/10.47197/retos.v37i37.69238

Ministerio de Desarrollo Social y Familia. (2020). Radiografía de la obesidad infantil. http://eligevivirsano.gob.cl/wpcontent/uploads/2020/01/PPT-EVS-15012020-Final.pdf

Ministerio de Educación. (2019). Plan de estudios para $3^{\circ}$ y $4^{\circ}$ medio. Formación general humanístico-científica, técnico-profesional y artística y formación diferenciada humanístico-científica. Acuerdo N057-2019 del 15 de mayo de 2019. https://curriculumnacional.mineduc.cl/614/articles-89597_recurso_11.pdf

Ministerio de Educación. (2020). Orientaciones para la actividad física escolar. https://convivenciaescolar.mineduc.cl/wp-content/uploads/2020/11/ORIENTACIONES-ED-FISICAWEB.pdf

Ministerio del Deporte. (2018). Encuesta nacional de hábitos de actividad física y deporte 2018 en población de 18 años y más. http://www.mindep.cl/encuesta-actividad-fisica-y-deporte-2018/ 
Ministerio del Deporte, Ministerio de Salud, \& Ministerio de Educación. (2017). Recomendaciones para la práctica de actividad física según curso de vida. http://selloseligevivirsano.cl/wp-content/uploads/2019/03/guia-derecomendaciones-af-curso-de-vida.pdf

Olmedo, J. (2000). Estrategias para aumentar el tiempo de práctica motriz en las clases de Educación Física escolar. Apunts. Educació Física L Esports, 59, 22-30. https://revista-apunts.com/estrategias-para-aumentar-eltiempo-de-practica-motriz-en-las-clases-de-educacion-fisica-escolar/

Organización de las Naciones Unidas para la Educación, la Ciencia y la Cultura. (2015). Educación física de calidad. Guía para los responsables políticos. https://unesdoc.unesco.org/ark:/48223/pf0000231340

Organización Mundial de la Salud. (2019). Plan de acción mundial sobre actividad física 2018-2030. Personas más activas para un mundo más sano. https://apps.who.int/iris/bitstream/handle/10665/327897/WHO-NMHPND-18.5-spa.pdf?sequence=1\&isAllowed=y

Organización Panamericana de la Salud. (2019). Directrices sobre la actividad física, el comportamiento sedentario y el sueño mara menores de 5 años. https://iris.paho.org/bitstream/handle/10665.2/51805/9789275321836_spa.pdf?sequence=5\&isAllowed=y

Pérez, P. (2018). Metodología y aplicación práctica de la biomecánica deportiva. Paidotribo.

Pieron, M. (1988). Didáctica de las actividades físicas y deportivas. Gymnos.

Retamal-Valderrama, C., Delgado-Floody, P., Espinoza-Silva, M., \& Jérez-Mayorga, D. (2019). Comportamiento del profesor, intensidad y tiempo efectivo de las clases de Educación Física en una escuela pública: Un acercamiento a la realidad. Retos, 35, 160-163. https://dialnet.unirioja.es/servlet/articulo?codigo=6761652

Reyes, A., Rivas, J., \& Pávez-Adasme, J. (2020). Tiempo de compromiso motor en la clase de educación física. Voces $\begin{array}{llll}\text { de la } & \text { Educación, } & \text { 90-113. }\end{array}$ https://www.revista.vocesdelaeducacion.com.mx/index.php/voces/article/view/256/213

Rodríguez, A. F., Rodríguez A., J. C., Guerrero G., H. I., Arias M., E. R., Paredes A., A. E., \& Chávez V., V. A. (2020). Beneficios de la actividad física para niños y adolescentes en el contexto escolar. Revista Cubana de Medicina General Integral, 36(2), e1535. http://scielo.sld.cu/pdf/mgi/v36n2/1561-3038-mgi-36-02e1535.pdf

Rodríguez, J., \& Terrados, N. (2006). Métodos para la valoración de actividad física y el gasto energético en niños y adultos. Archivos de Medicina del Deporte, 23(115), 365-377. https://archivosdemedicinadeldeporte.com/articulo/en/65/2001/729/

Rodríguez-Rodríguez, F., Curilem G., C., Escobar G., D., \& Valenzuela E., L. (2016). Propuesta de evaluación de la educación física escolar en Chile. Educación Física y Ciencia, 18(1), e003. http://www.efyc.fahce.unlp.edu.ar/article/view/EFyCv18n01a03/

Ruiz, C., Lara, A., López, F., Cachón, J., \& Valdivia, P. (2019). Análisis del tiempo de clase en EF y propuestas para su optimización. Retos, 35, 126-129. https://recyt.fecyt.es/index.php/retos/article/view/61880/40841

Salas, C., Peterman, F., Martínez, M., Leiva, A., Díaz, X., Garrido, A., Poblete-Valderrama, F., \& Celis-Morales, C. (2018a). Poco es mejor que nada: practicar actividad física de manera regular podría reducir a la mitad el riesgo de desarrollar diabetes mellitus. Revista Médica Clínica Las Condes, 29(1), 98-100. https://doi.org/10.1016/j.rmclc.2017.11.009 
Salas, C., Peterman-Rocha, F., Celis-Morales, C., \& Martínez-López, E. J. (2018b). Apoyo parental para realizar actividad física en escolares de 6 años de edad: influencia sobre el estado nutricional y fitness. Revista Chilena de Pediatría, 89(6), 732-740. https://doi.org/10.4067/S0370-41062018005000906

Salazar, C., Flores, P., Barajas, L., Medina, R., Chávez, M., \& Camacho, E. (2017). Evaluación del tiempo efectivo de la clase de educación física en escolares del ámbito rural. Revista Edu-fisica.com, 9(19), 34-46. http://revistas.ut.edu.co/index.php/edufisica/article/view/1097

Sallis, J., McKenzie, T, \& Alcaráz, F. (1993). Habitual physical activity and elath-related physical fitness in fourthgrade children. American Journal of Deseases in Childhood, 147, 890-896. https://doi.org/10.1001/archpedi.1993.02160320092025

Sánchez-Meca, J., \& Botella, J. (2010). Revisiones sistemáticas y meta-análisis: herramientas para la práctica profesional. Papeles del Psicólogo, 31(1), 7-17. http://www.papelesdelpsicologo.es/pdf/1792.pdf

Scruggs, P., Beveridge, S., Eisenman, P., Watson, D., Shultz, B., \& Ransdell, L. (2003). Quantifying physical activity via pedometry in elementary physical education. Medicine y Science in Sports y Exercise, 35(6), 1065-1071. https://doi.org/10.1249/01.MSS.0000069748.02525.B2

Siedentop, D. (1998). Aprender a enseñar la Educación Física. INDE.

Smith, N., Lounsbery, M., \& McKenzie, T. (2014). Physical activity in high school physical education: Impact of lesson context and class gender composition. Journal of Physical Activity and Health, 11(1), 127-135. https://doi.org/10.1123/jpah.2011-0334

Sutherland, R., Campbell, E., Lubans, D. R., Morgan, P. J., Okely, A. D., Nathan, N., Gillham, K., Lecathelinais, C., \& Wiggers, J. (2016). Physical education in secondary schools located in low-income communities: physical activity levels, lesson context and teacher interaction. Journal of Science and Medicine in Sport, 19(2), 135141. https://doi.org/10.1016/j.jsams.2014.12.003

Telama, R. (2009). Tracking of physical activity from childhood to adulthood: a review. Obesity Facts, 2(3), 187-195. https://doi.org/10.1159/000222244

Torres, L. J. (2011). Caracterización de la utilización del tiempo en las clases de educación física en las escuelas del Consejo Popular Guáimaro Norte del municipio Guáimaro. Revista EFDeportes, 16(156). https://www.efdeportes.com/efd156/utilizacion-del-tiempo-en-educacion-fisica.htm

Trejo, P. M., Jasso C., S., Mollinedo M., F. E., \& Lugo B., L. G. (2012). Relación entre actividad física y obesidad en escolares. Revista Cubana de Medicina General Integral, 28(1), 34-41. http://scielo.sld.cu/pdf/mgi/v28n1/mgi05112.pdf

Universidad de la Frontera, \& Active Healthy Kids Global Alliance. (2018). Reporte de Notas de Actividad Física Infantil 2018 Chile. http://www.activehealthykidschile.com/wp-content/uploads/2019/04/Reporte-de-NotasDiptico-2018-v2-hojas.pdf

Urrútia, G., \& Bonfill, X. (2010). Declaración PRISMA: una propuesta para mejorar la publicación de revisiones sistemáticas y metaanálisis. Medicina Clínica, 135(11), 507-511. https://doi.org/10.1016/j.medcli.2010.01.015 
Vio del Río, F. (2019, 30 de mayo). Pérdida de obligatoriedad de las clases de educación física en tercero y cuarto año medio. Instituto de Nutrición y Tecnología de los Alimentos. https://inta.cl/perdida-de-obligatoriedad-de-lasclases-de-educacion-fisica-en-tercero-y-cuarto-ano-medio

Weinberg, R., \& Gould, D. (1995). Foundations of sport and excercise psycology. Human Kinetics.

Yanci I., J., Vinuesa M., A., Rodríguez N., J., \& Yanci I., L. (2016). El tiempo de compromiso motor en las sesiones de educación física del primer y segundo ciclo de educación primaria. Sportis, Revista Técnico-Científica Del Deporte Escolar, Educación Física y Psicomotricidad, 2(2), 239-253. https://doi.org/10.17979/sportis.2016.2.2.1447

\section{Nota}

Este artículo fue realizado como Trabajo Final de Grado de la carrera de Pedagogía en Educación Física en la Universidad Adventista de Chile, Chillán, Chile.

\section{Cómo citar}

Reyes Rodríguez, A., Ibañez Alarcón, M., Villagra, N., Maureira, P., \& Pávez-Adasme, G. (2021). Tiempo de compromiso motor en educación física para enseñanza primaria. Una revisión sistemática. Páginas de Educación, 14(2), 01-27. https://doi.org/10.22235/pe.v14i2.2587

\section{Contribución autoral}

a) Concepción y diseño del trabajo; b) Adquisición de datos; c) Análisis e interpretación de datos; d) Redacción del manuscrito; e) revisión crítica del manuscrito.

A. R. R. ha contribuido en a, b, c, d, e; M. I. A. en b, c, d, e; N. V. en b, c, d, e; P. M. en b, c, d, e y G. P.-A. en a, c, d, e.

Editora científica responsable: Mag. Florencia de León 